\title{
Laryngeal mask airway and the enigma of anatomical sizing
}

\author{
Davide Cattano $^{1}$ • Tom C. R. V. Van Zundert ${ }^{2} \cdot$ Jacek Wojtczak ${ }^{3}$
}

Received: 18 April 2019 / Accepted: 22 April 2019 / Published online: 24 April 2019

(c) Springer Nature B.V. 2019

The laryngeal mask airway (LMA, Teleflex Medical ${ }^{\circledR}$, Westmead, Ireland) is a device that has proved itself over the last 30 years in a large group of patients undergoing diagnostic procedures or interventional surgery. Laryngeal mask airways (LMAs) and more in general supraglottic airway devices or broadly defined as extraglottic airway devices (EADs), are safe and effective airway devices: designed with a specific purpose, being utilized to provide adequate gas exchange and prevent airway obstruction during general anesthesia without the use of an endotracheal tube. EADs are useful as primary (first airway), secondary (rescue of a difficult airway) and tertiary (as conduit for scopes) tools in airway management $[1,2]$.

While the use of an EAD involves a standardized sizing based on manufactured recommendations, the device sizing has been a long-time font of academic research and arguments $[3,4]$. Two of the co-authors (DC, TVZ) of this editorial had the chance to work on such debated topic in their $\mathrm{PhD}$-dissertations ('Selection of the proper LMA size: gender or weight based?' UniCampus, LUCBM, Rome, Italy, 1999 and 'Improvements towards safer extraglottic airway devices. University of Maastricht, The Netherlands, 2015). It does result that such issue may be common across several other EADs. Moreover, the correct sizing and recognition of factors influencing the effective and safe use of an LMA or EADs, seems critical in normal but even more in difficult airways [5].

It turns out that common anatomical factors and measurement, typically and readily available to be assessed, are differently associated with laryngeal anatomy distances and

Davide Cattano

Davide.Cattano@uth.tmc.edu

1 Department of Anesthesiology, The McGovern Medical School at Houston, UTHealth, Houston, TX, USA

2 Department of Anesthesiology, Ziekenhuis Oost Limburg, Genk, Belgium and Udayana University, Bali, Indonesia

3 Department of Anesthesiology, The University of Rochester, Rochester, NY, USA dimensions [6, 7] As well, the design of LMA was based on a generalized cast acquired in few subjects (cadavers): while it is true that subsequent studies used radiological information and clinical usage has confirmed the effectiveness of the LMA design, yet limitations of MRI and CT scan are well known [8-10].

In this issue of the Journal of Clinical Monitoring Zhue et al. [11] published an interesting study presenting a new method for the size-selection of the Classic LMA. In 74 patients before the induction of general anesthesia, the authors measured the linear distance between the cricoid cartilage and the mentum (C-M distance). The $\mathrm{C}-\mathrm{M}$ distance reflects the length of the pharyngeal cavity and therefore, the CM-based size selection of the LMA provided a better airway seal and higher success rate of the first insertions than the weight-based method. This study confirms the hypothesis that the external neck landmarks are more useful in the LMA size selection than the weight-based method $[6,7,12]$ Why only the LMA-Classic was studied and whether other EADs may be following the same rule remains a speculation, yet some of the reasons can be assumed in the considerations to follow.

In a recent study it was showed that the external neck measurements overestimate the internal measurements which can also be assessed by the ultrasound (US) exam [6, $10,12]$. The US proved to visualize the thyroid and arytenoid cartilages in control conditions and after the placement of the LMA [13, 14]. Likewise, such findings have been confirmed in a pediatric population as well: this is quite important especially in consideration of the view-changes from the traditional anatomy differences assumed and taught over previous years between adult and pediatric patients [15]. Indeed, US was able to detect the malposition of the LMA.

Based on Zhu et al. report [11], we are eager to assume that traditional strategies to use weight (considered as ideal body weight respective to height group) has proved effective (performing with adequate safety margins). Yet, other strategies may offer more accurate sizing and fitting of the LMA (maybe other EADs as well): such methods may result 
particularly and possibly better, when the values are outside normal ranges (extreme weights or ages).

It is also legitimate to think that when considering the variety of devices' design, as much as they can equally perform, has made researcher willing to look into other ways of estimating best-fit sizing and possibly even assigning different devices based on such anatomical landmarks. Additional thoughts about the study underscore a limitation and pertain the use of neuromuscular blockade: so the results might not be extrapolated to patients undergoing spontaneous respiration because hypothetically the hypopharyngeal muscle tension may affect LMA positioning. Another consideration pertains the CM distance: while it is indeed important, it does not differentiate between a patient with a large (obstructive) tongue and a patient with a normal size tongue. It is the space behind the tongue-distance back size of the tongue till the posterior pharyngeal wall - that may be important to be able to successfully insert the EAD behind the tongue into the hypopharynx.

In summary, Zhu et al. add another important evidence to support the use of US: a bedside, point of care examination potentially could finally offers an objective tool to size the best selection method for an EAD. Whether this will solve the issue of correct EADs sizing still needs to be proven in further studies.

\section{Compliance with ethical standards}

Conflict of interest All authors declare that they have no conflict of interest.

\section{References}

1. van Zundert TC, Brimacombe JR, Ferson DZ, Bacon DR, Wilkinson DJ. Archie brain: celebrating 30 years of development in laryngeal mask airways. Anaesthesia. 2012;67:1375-85.

2. Gordon J, Cooper RM, Parotto M. Supraglottic airway devices: indications, contraindications and management. Minerva Anestesiol. 2018;84:389-97.

3. Van Zundert TC, Hagberg CA, Cattano D. Inconsistent size nomenclature in extraglottic airway devices. Minerva Anestesiol. 2014;80:692-700.
4. van Zundert TC, Hagberg CA, Cattano D. Standardization of extraglottic airway devices, is it time yet? Anesth Analg. 2013;117:750-2.

5. Katsiampoura AD, Killoran PV, Corso RM, Cai C, Hagberg CA, Cattano D. Laryngeal mask placement in a teaching institution: analysis of difficult placements. F1000Res. 2015;4:102.

6. Cattano D, Wojtczak JA, Callender R, Cai C, Tezino T, van Zundert TCRV, et al. External neck landmark identification and measurement correlation in a normal weight cohort. J Anesthesiol Clin Sci. 2014;3:1-6.

7. Cattano D, Van Zundert Zundert TC, Wojtzack J, Cai C, Callender R, Marjiya SE, Hagberg C. A new method to test concordance between extraglottic airway device dimensions and patient anatomy. Anesthesiology. 2014;A3148.

8. Russo SG, Cremer S, Eich C, Jipp M, Cohnen J, Strack M, Quintel $\mathrm{M}$, Mohr A. Magnetic resonance imaging study of the in vivo position of the extraglottic airway devices i-gel ${ }^{\mathrm{TM}}$ and LMASupreme ${ }^{\mathrm{TM}}$ in anaesthetized human volunteers. Br J Anaesth. 2012;109:996-1004.

9. Ucisik-Keser FE, Chi TL, Hamid Y, Dinh A, Chang E, Ferson DZ. Impact of airway management strategies on magnetic resonance image quality. Br J Anaesth. 2016;117(Suppl 1):i97-102.

10. Cattano D, Lee S, Corso RM, Hagberg CA, Wojtczak JA. Comparison of internal neck anatomy with extraglottic airway device dimensions using radiographic imaging. Anesthesiology. 2016; A1141.

11. Zhu Y, Shen W, Lin Y, Huang T, Xie L, et al. Cricoid-mental distance-based versus weight-based criteria for size selection of classic laryngeal mask airway in adults: A Randomized Controlled Study. J Clin Monit Comput. 2019. https://doi.org/10.1007/s1087 7-019-00308-w.

12. Lee SM, Wojtczak JA, Cattano D. Ultrasound comparison of external and internal neck anatomy with the LMA Unique. J Ultrason. 2017;17:229-34.

13. Corso RM, Lee S, Wojtczak J, Cattano D. Extraglottic airway devices and ultrasound airway evaluation: are we on the right track? Minerva Anestesiol. 2017;83:1110-1.

14. Wojtczak JA, Cattano D. Laryngo-tracheal ultrasonography to confirm correct endotracheal tube and laryngeal mask airway placement. J Ultrason. 2014;14:362-6.

15. Kim J, Kim JY, Kim WO, Kil HK. An ultrasound evaluation of laryngeal mask airway position in pediatric patients: an observational study. Anesth Analg. 2015;120:427-32.

Publisher's Note Springer Nature remains neutral with regard to jurisdictional claims in published maps and institutional affiliations. 